\title{
NOTE \\ Characterization of a Rothia-like organism from a mouse: description of Rothia nasimurium sp. nov. and reclassification of Stomatococcus mucilaginosus as Rothia mucilaginosa comb. nov.
}

\author{
M. D. Collins, ${ }^{1}$ R. A. Hutson, ${ }^{1}$ V. Båverud ${ }^{2}$ and E. Falsen ${ }^{3}$
}

Author for correspondence: M. D. Collins. Tel: +44 1189357226 . Fax: +44 1189267917. e-mail:m.d.collins@reading.ac.uk

\footnotetext{
1 Department of Food Science and Technology, University of Reading, Reading RG6 6AP, UK

2 National Veterinary Institute, S-75007 Uppsala, Sweden

3 Culture Collection, Department of Clinical Bacteriology, University of Göteborg, Sweden
}

\begin{abstract}
An unknown, Gram-positive, ovoid-shaped bacterium isolated from the nose of a mouse was subjected to a polyphasic taxonomic analysis. Comparative 165 rRNA gene sequencing demonstrated that the unknown organism was a member of the family Micrococcaceae and possessed a specific phylogenetic association with Rothia dentocariosa and Stomatococcus mucilaginosus. Phenotypically, the bacterium closely resembled $R$. dentocariosa and $S$. mucilaginosus but could be distinguished from these species by biochemical tests and electrophoretic analysis of whole-cell proteins. Based on both phylogenetic and phenotypic evidence, it is proposed that the unknown bacterium be classified in the genus Rothia, as Rothia nasimurium sp. nov. In addition, it is proposed that $S$. mucilaginosus be reclassified in the genus Rothia, as Rothia mucilaginosa comb. nov.
\end{abstract}

Keywords: Rothia, Stomatococcus, taxonomy, phylogeny, 16S rRNA

Rothia dentocariosa is found in the oral cavity and pharynx of man where it forms part of the normal microflora (Schaal, 1992). This species is also now recognized as an opportunistic pathogen causing septicaemia and endocarditis, as well as other serious infections (e.g. Schafer et al., 1979; Pape et al., 1979; Pers et al., 1987; Minato \& Abiko, 1984; Schiff \& Kaplan, 1987). The taxonomic affinities of $R$. dentocariosa have always been controversial. Primarily because of its cellular morphology, the species has historically been associated with Actinomyces and related genera (Schaal, 1992). In recent years it has become apparent, however, that it is only remotely related to these taxa, and is phylogenetically a member of the family Micrococcaceae (Stackebrandt et al., 1997), exhibiting a specific association with Stomatococcus mucilaginosus. Like $R$. dentocariosa, S. mucilaginosus is a resident of the human oral cavity and pharynx. $R$. dentocariosa is presently the only recognized species of the genus Rothia. There is, however, a considerable body of evidence indicating some bio-

The GenBank accession number for the 16S rRNA sequence of Rothia nasimurium CCUG $35957^{\top}$ is AJ 131121. chemical and serological heterogeneity within the species (e.g. Lesher et al., 1974; Schofield \& Schaal, 1981; Fotos et al., 1984; Kronvall et al., 1998). In addition, the existence of a second genomovar of $R$. dentocariosa has recently been described from humans (Kronvall et al., 1998). It is currently not known whether $R$. dentocariosa or similar organisms occur in other animal species (Schaal, 1992). During the course of a study of unusual Actinobacteria from animals, we have characterized a novel Gram-positive, facultatively anaerobic coccus from the nose of a healthy mouse which phenotypically resembles the genus Rothia. Based on the results of a polyphasic taxonomic study, we propose a new species, Rothia nasimurium, for this bacterium.

Strain CCUG $35957^{\mathrm{T}}$ was isolated from the nose of a healthy mouse. The unidentified organism was cultured on Columbia blood agar base supplemented with $5 \%$ defribrinated horse blood at $37^{\circ} \mathrm{C}$ in air plus $5 \% \mathrm{CO}_{2}$. The strain was biochemically characterized by using the API rapid ID32 STREP, API CORYNE and API ZYM systems according to the manufacturer's instructions (API bioMérieux). Preparation of cellular protein extracts for PAGE analysis, densito- 


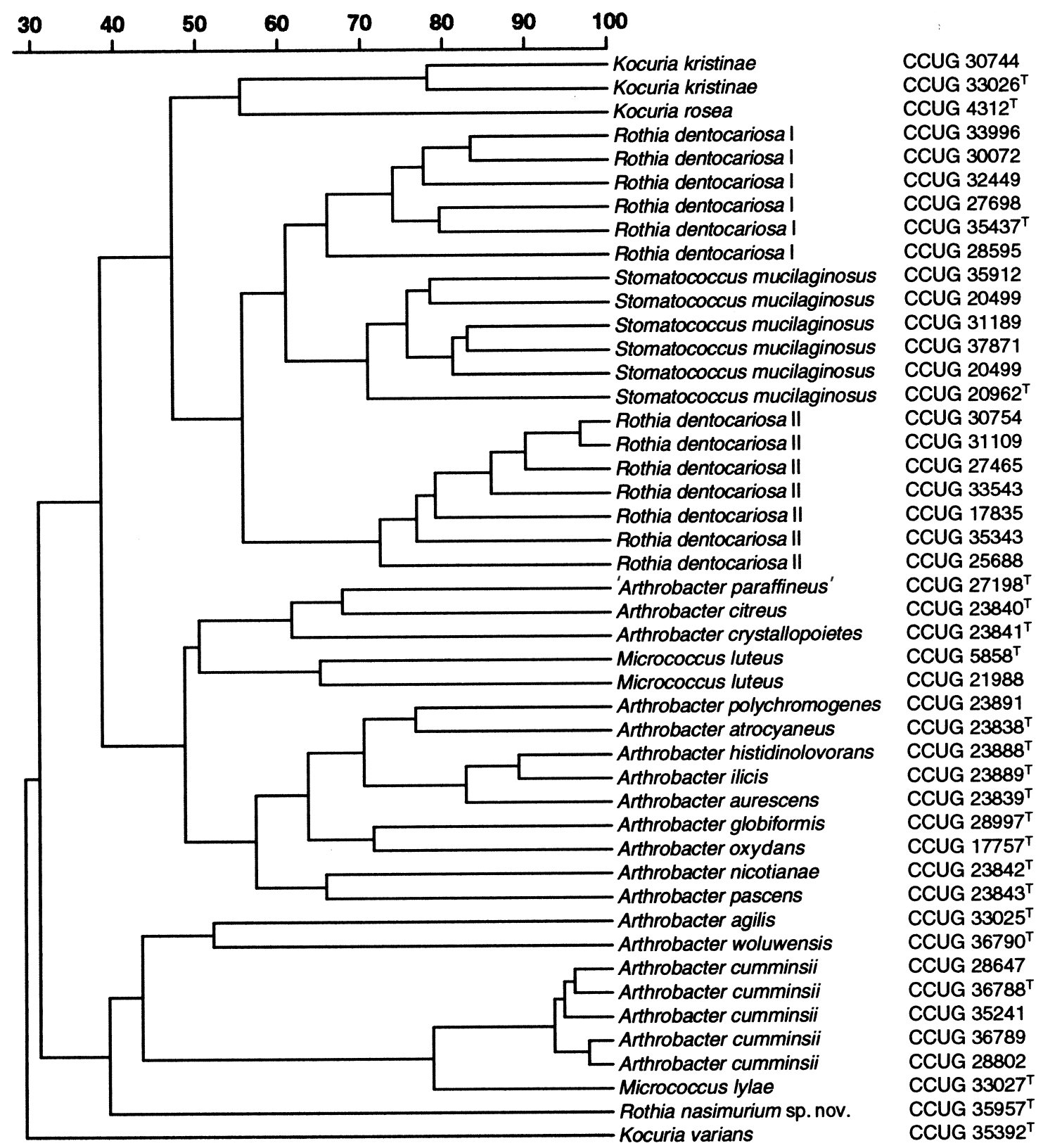

Fig. 1. Dendogram derived from the unweighted pair group average linkage of correlation coefficients (expressed as percentage values) between whole-cell protein patterns of $R$. nasimurium sp. nov. and some relatives. Duplicate cultures of strain CCUG 20499 were run to assess reproducibility.

metric analysis, normalization of the protein profiles and numerical analyses were performed as described by Pot et al. (1994) using the GelCompar 3.0 software package (Applied Maths). The similarity between all pairs of traces was expressed by the Pearson product moment correlation coefficient, converted for convenience to a percentage similarity value. Long-chain cellular fatty acids were examined using the MIDI system. Cells for fatty acid analysis were prepared on blood agar at $37^{\circ} \mathrm{C}$. The DNA $\mathrm{G}+\mathrm{C}$ content was determined by thermal denaturation as described by Garvie (1978). The 16S rRNA genes of the isolate were amplified by PCR and directly sequenced using a Taq DyeDeoxy Terminator Cycle Sequencing kit (Applied Biosystems) and an automatic DNA sequencer (model 373A, Applied Biosystems). The closest known relatives of the new isolate were determined by performing database searches. These sequences and those of other known related strains were retrieved from the GenBank or Ribosomal Database Project (RDP) libraries and aligned with the newly determined sequence using the program PILEUP (Devereux et al., 1984). The resulting multiple sequence alignment was corrected manually and a distance matrix was calcu- 
lated using the programs PRETTY and DNADIST (using the Kimura-2 correction parameter) (Felsenstein, 1989). A phylogenetic tree was constructed according to the neighbour-joining method with the program NEIGHBOR (Felsenstein, 1989). The stability of the groupings was estimated by bootstrap analysis ( 500 replications) using the programs DNABOOT, DNADIST, NEIGHBOR and CONSENSE (Felsenstein, 1989). The GenBank accession number of the 16S rRNA gene sequence of strain CCUG $35957^{\mathrm{T}}$ is AJ 131121.

The unidentified isolate consisted of Gram-positive, non-motile, ovoid-shaped cells. The organism grew on blood agar producing a weak $\alpha$-haemolytic reaction and was non-pigmented. It was facultatively anaerobic and catalase-positive. Using commercially available API systems, the isolate produced acid from glucose, lactose, maltose, methyl $\beta$-D-glucopyranoside, sucrose and trehalose, but failed to produce acid from Larabinose, D-arabitol, cyclodextrin, glycogen, mannitol, melibiose, melezitose, $N$-acetylglucosamine, pullulan, raffinose, ribose, sorbitol, tagatose or Dxylose. Reactions for alanine phenylalanine proline arylamidase, cystine arylamidase, ester lipase C-8, $\beta$ galacturonidase, $\alpha$-glucosidase, leucine arylamidase, valine arylamidase, pyrazinamidase and trypsin were positive, whereas those for alkaline phosphatase, arginine dihydrolase, esterase $\mathrm{C}$ - $4, \alpha$-fucosidase, $\alpha$ galactosidase, $\beta$-glucosidase, $\beta$-glucuronidase, lipase $\mathrm{C} 14, \alpha$-mannosidase, $\beta$-mannosidase and urease were negative. The organism hydrolysed aesculin, reduced nitrate and was Voges-Proskauer-negative. In terms of its overall biochemical characteristics, the unidentified bacterium closely resembled $R$. dentocariosa and $S$. mucilaginosus. However, it differed from these species in producing acid from lactose and by its positive reactions for valine arylamidase, ester lipase C-8 (weak reaction) and trypsin (weak reaction). By contrast, $R$. dentocariosa (genomovar I and II) and $S$. mucilaginosus do not produce acid from lactose and are negative for these enzymes. An examination of the long-chain cellular fatty acids of the isolate revealed acids predominantly of the iso and anteiso methylbranched types (composition: ai-C13, $2.0 \%$; i-C14, 7.7 $\%$; C14:0, $1 \cdot 3 \%$; i-C15, 5.1\%; ai-C15, 56.8\%; C15:0, $0.5 \%$; i-C16, $18.7 \%$; C16:0, 2.5\%; ai-C17, 5.4\%). Determination of the fatty acid compositions of $R$. dentocariosa CCUG $35437^{\mathrm{T}}$ (composition: i-C14, $4.0 \%$; i-C15, $3 \cdot 3 \%$; ai-C15, 53.3\% ; C15:0, 2.2\% ; i$\mathrm{C} 16,14.6 \%$; $\mathrm{C} 16: 0,6.0 \%$; ai-C17, 16.9\%), R. dentocariosa genomovar II CCUG 17835 (composition: i$\mathrm{C} 14,3.5 \%$; i-C15, $4.5 \%$; ai-C15, $51.6 \%$; i-C16, $17 \cdot 3 \%$; C16:0, 7.1\%; ai-C17, 16.0\%) and S. mucilaginosus CCUG 20962 (composition: i-C14, 10.8\% ; i-C15, 5.9\% ; ai-C15, 45.7\% ; i-C16, $21.6 \%$; C16:0, $9.6 \%$; ai-C17, $6.4 \%$ ) grown under similar conditions revealed very similar profiles to the unknown isolate, thereby reinforcing the affinity between these taxa. PAGE analysis of whole-cell protein patterns, however, further demonstrated that the unidentified organism was phenotypically distinct from both $R$. dentocariosa and S. mucilaginosus (Fig. 1). To ascertain the phylogenetic position of the unknown isolate, its almost complete $16 \mathrm{~S}$ rRNA gene sequence was determined and subjected to a comparative analysis. Sequence database searches revealed that the unknown bacterium was phylogenetically a member of the Actinobacteria and displayed a specific association with members of the family Micrococcaceae (Stackebrandt et al., 1997). Highest sequence similarity was shown with $R$. dentocariosa $(96.6 \%), S$. mucilaginosus $(96.6 \%)$, Kocuria erythromyxa (96.6\%), Kocuria rosea $(96 \cdot 6 \%)$, Kocuria kristinae $(95 \cdot 3 \%)$, Kocuria palustris $(95.5 \%)$, with other members of the Micrococcaceae displaying somewhat lower levels of relatedness (approx. 92-95\%; data not shown). A tree constructed by the neighbour-joining method depicting the phylogenetic relationships of the unidentified organism is shown in Fig. 2 and unequivocally demonstrates that it represents a new species within the Micrococcaceae. The bacterium formed a distinct line branching from the base of a cluster which embraced $R$. dentocariosa (genomovar I and II) and $S$. mucilaginosus.

The polyphasic taxonomic analysis has shown that the unknown ovoid-shaped bacterium isolated from the nose of a mouse represents a hitherto unrecognized species within the Micrococcaceae. Phylogenetically, the bacterium forms a distinct subline and exhibits a specific association with the Rothia-Stomatococcus group of organisms. The clustering of the unknown mouse bacterium with this latter group was supported by a bootstrap resampling value of $100 \%$ (Fig. 2). The close relationship between the unknown organism and Rothia-Stomatococcus is supported by their close biochemical resemblance. Based on phylogenetic and phenotypic evidence, the unknown bacterium clearly merits classification as a new species, for which Rothia is the most appropriate genus. It has long been recognized that $R$. dentocariosa is phenotypically heterogeneous (Lesher et al., 1974; Schofield \& Schaal, 1981; Fotos et al., 1984), raising the possibility of a second species within the genus Rothia. Indeed, a recent study using protein profiling and 16S rRNA gene sequencing has resulted in the recognition of a second species, designated $R$. dentocariosa genomovar II (Kronvall et al., 1980). The latter genomovar has, however, not been formally named as it is not possible to biochemically distinguish it from authentic $R$. dentocariosa. Thus, given the distinct biochemical characteristics of the new murine isolate, the organism reported here should not be confused with earlier reports of variants within $R$. dentocariosa. In addition to biochemical differences, the murine bacterium also differs from $R$. dentocariosa strains ( both genomovars I and II) in whole-cell protein profiles and by displaying $>3 \% 16 \mathrm{~S}$ rRNA sequence divergence. It is pertinent to note that $S$. mucilaginosus displays a closer phylogenetic affinity to $R$. dentocariosa than to the unknown bacterium. Hence the classification of the new mouse bacterium within the genus Rothia also 


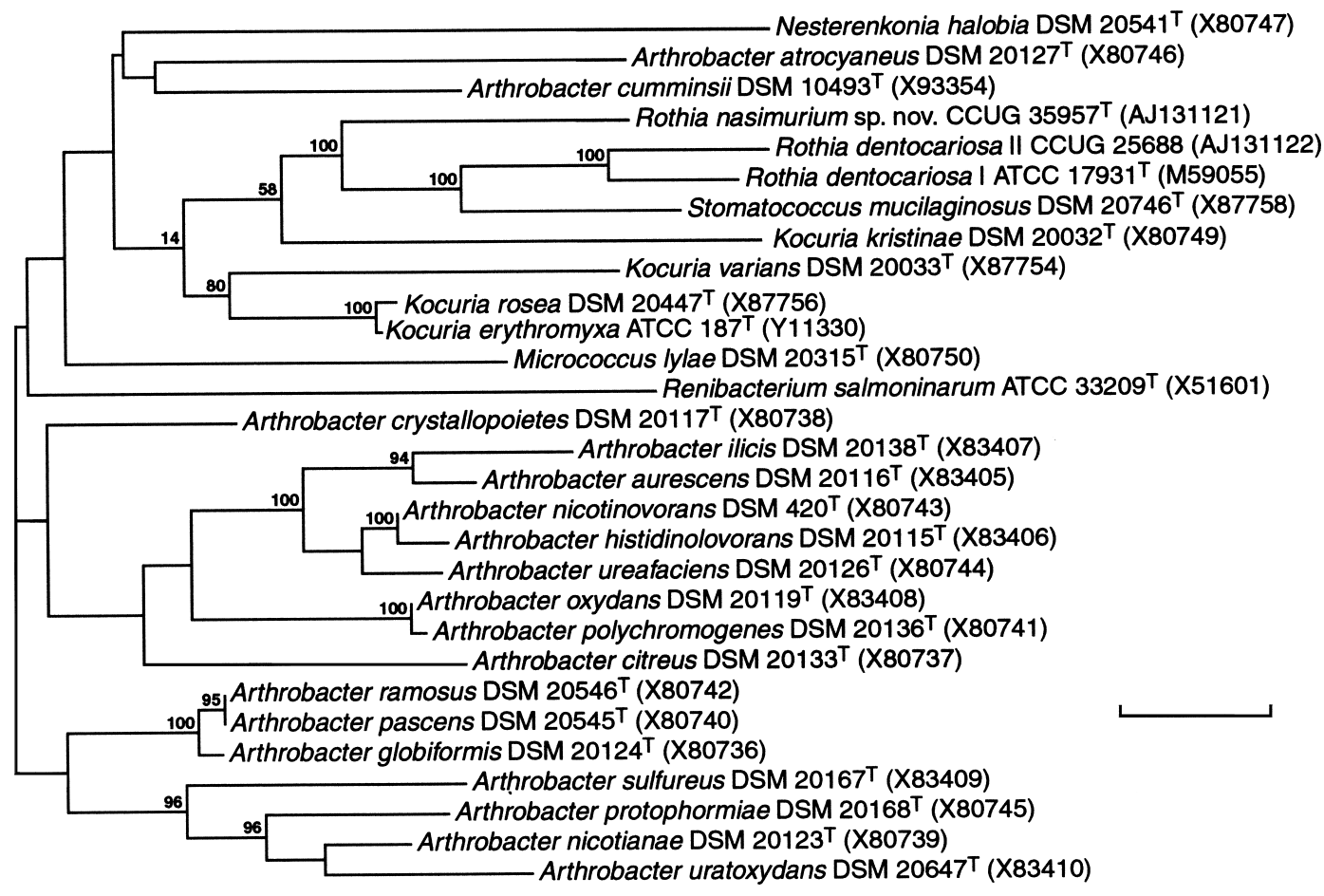

Fig. 2. Unrooted tree based on $16 \mathrm{~S}$ rRNA analysis showing the phylogenetic relationships of $R$. nasimurium $\mathrm{sp}$. nov. The bar represents $1 \%$ sequence divergence.

necessitates the assignment of $S$. mucilaginosus to this genus. We consider that the case for reclassifying $S$. mucilaginosus in the genus Rothia is very strong given their close biochemical similarity, and also on phylogenetic grounds (tree topology considerations and 16S rRNA sequence divergence values). Support for the close relationship between Rothia and Stomatococcus is also evident from PAGE analysis of whole-cell proteins. Indeed, based on whole-cell protein patterns, it is apparent that $S$. mucilaginosus phenotypically more closely resembles $R$. dentocariosa genomovar I (which includes the type strain of the species) than does $R$. dentocariosa genomovar II (Fig. 1; see also Kronvall et al., 1998). Therefore, based on both phylogenetic and phenotypic evidence, we propose the unknown mouse bacterium be classified in the genus Rothia (Georg and Brown 1967), as Rothia nasimurium sp. nov. In addition, we propose the reclassification of $S$. mucilaginosus (Bergan and Kocur 1982) in the genus Rothia, as Rothia mucilaginosa comb. nov.

\section{Description of Rothia nasimurium sp. nov.}

Rothia nasimurium (na.si.mu'ri.um. M.L. pl. gen. n. nasimurium of the nose of mice).

Cells are Gram-positive, non-motile cocci. Produce a weak $\alpha$-haemolysis on blood agar. Non-pigmented. Facultatively anaerobic and catalase-positive. Acid is produced from glucose, lactose, maltose, methyl $\beta$-Dglucopyranoside, sucrose and trehalose. Acid is not produced from L-arabinose, D-arabitol, cyclodextrin, glycogen, mannitol, melibiose, melezitose, $N$-acetylglucosamine, pullulan, raffinose, ribose, sorbitol, tagatose or D-xylose. Alanine phenylalanine proline arylamidase, cystine arylamidase, ester lipase $\mathrm{C}-8, \beta$ galacturonidase, $\alpha$-glucosidase, leucine arylamidase, valine arylamidase, pyrazinamidase and trypsin are produced. Alkaline phosphatase, arginine dihydrolase, esterase $\mathrm{C}$ - $4, \alpha$-fucosidase, $\alpha$-galactosidase, $\beta$-glucosidase, $\beta$-glucuronidase, lipase C14, $\alpha$-mannosidase, $\beta$ mannosidase, pyrrolydonyl arylamidase and urease are not produced. Acid phosphatase, chymotrypsin, pyroglutamic acid arylamidase, $\beta$-galactosidase, glycine tryptophan arylamidase may or may not be produced. Aesculin is hydrolysed but not hippurate. Nitrate is reduced. Voges-Proskauer-negative. Cellular fatty acids are mainly of the methyl-branched types with ai-C15 predominating. Isolated from the nose of a healthy mouse. Habitat unknown. The $\mathrm{G}+\mathrm{C}$ content of the DNA is $56 \mathrm{~mol} \%$. The type strain is CCUG $35957^{\mathrm{T}}$.

\section{Description of Rothia mucilaginosa (Bergan and Kocur 1982) comb. nov.}

Rothia mucilaginosa (mu.ci.la.gi.no'sa. L. adj. mucilaginosa slimy).

The description of Rothia mucilaginosa is as given for Stomatococcus mucilaginosa by Bergan \& Kocur (1982). 


\section{Acknowledgements}

We are grateful to Professor Hans Trüper (University of Bonn, Germany) for help in coining the species epithet, and to Berit Sjödén and Maria Ohlén for processing of strains.

\section{References}

Bergan, T. \& Kocur, M. (1982). Stomatococcus mucilaginosus gen. nov., sp. nov., ep. rev., a member of the family Micrococcaceae. Int J Syst Bacteriol 32, 374-377.

Devereux, J., Haeberli, P. \& Smithies, O. (1984). A comprehensive set of sequence analysis programs for the VAX. Nucleic Acids Res 12, 387-395.

Felsenstein, J. (1989). PHYLIP - phylogeny inference package (version 3.2). Cladistics 5, 164-166.

Fotos, P. G., Gerencser, M. A. \& Yelton, D. B. (1984). Strain differentiation of Rothia dentocariosa and related isolates by sodium dodecyl sulfate-polyacrylamide gel electrophoresis. Int J Syst Bacteriol 34, 102-106.

Garvie, E. I. (1978). Streptococcus raffinolactis (Orla-Jensen and Hansen); a group $\mathrm{N}$ streptococcus found in raw milk. Int $J$ Syst Bacteriol 28, 190-193.

Georg, L. K. \& Brown, J. M. (1967). Rothia gen. nov., an aerobic genus of the family Actinomycetaceae. Int J Syst Bacteriol 17, 79-88.

Kronvall, G., Lannér-Sjöberg, M., von Stedingk, L. V., Hanson, H. S., Petterson, B. \& Falsen, E. (1998). Whole-cell protein and partial 16S rRNA sequence analysis suggest the existence of a second Rothia species. Clin Microbiol Infect 4, 255-263.

Lesher, R. J., Gerenser, M. A. \& Gerenser, V. F. (1974). Morphological, biochemical, and serological characterization of Rothia dentocariosa. Int J Syst Bacteriol 24, 154-159.
Minato, K. \& Abiko, Y. (1984). $\beta$-Lactam antibiotic resistant Rothia dentocariosa from infected post-operative maxillary cyst: studies on R-plasmid and $\beta$-lactamase. Gen Pharmocol 15, 287-292.

Pape, J., Singer, C., Krehn, T. E., Lee, B. J. \& Armstrong, D. (1979). Infective endocarditis caused by Rothia dentocariosa. Ann Intern Med 91, 746-747.

Pers, C., Kristiansen, J. E., Joensson, V. \& Hansen, N. E. (1987). Rothia dentocariosa septicaemia in a patient with chronic lymphocytic leukaemia and toxic granulocytopenia. Dan Med Bull 34, 322-323.

Pot, B., Vandamme, P. \& Kersters, K. (1994). Analysis of electrophoretic whole-organism protein fingerprints. In Chemical Methods in Prokaryotic Systematics, pp. 493-521. Edited by M. Goodfellow \& A. G. O'Donnell. Chichester: Wiley.

Schaal, K. P. (1992). The genera Actinomyces, Arcanobacterium, and Rothia. In The Prokaryotes, 2nd edn, pp. 850-905. Edited by A. Balows, H. G. Trüper, M. Dworkin, W. Harder \& K.-H. Schleifer. New York: Springer.

Schafer, F. J., Wing, E. J. \& Norden, C. W. (1979). Infectious endocarditis caused by Rothia dentocariosa. Ann Intern Med $\mathbf{9 1}$, 747-748.

Schiff, M. J. \& Kaplan, M. H. (1987). Rothia dentocariosa pneumonia in an immunocompromised patient. Lung 165, 279-282.

Schofield, G. M. \& Schaal, K. P. (1981). A numerical taxonomic study of members of the Actinomycetaceae and related taxa. J Gen Microbiol 127, 237-259.

Stackebrandt, E., Rainey, F. A. \& Ward-Rainey, N. L. (1997). Proposal for a new hierarchic classification system, Actinobacteria classis nov. Int J Syst Bacteriol 47, 479-491. 